\title{
IEEE 802.15.3d-Compliant Waveforms for Terahertz Wireless Communications
}

\author{
Mohamed Shehata, Senior Member, IEEE, Ke Wang, Senior Member, IEEE, Julian Webber, Senior \\ Member, IEEE, Masayuki Fujita, Member, IEEE, Tadao Nagatsuma, Fellow, IEEE, \\ and Withawat Withayachumnankul, Senior Member, IEEE
}

\begin{abstract}
The terahertz electromagnetic band has been foreseen as a promising candidate to accommodate the everincreasing wireless data traffic. To this end, the development of novel transceiver architectures and signal processing solutions is crucial to handling massive data volume transmitted over terahertz wireless communications networks. In this paper, we propose a waveform that shows full compliance with the spectral emission mask imposed by the IEEE Standard for terahertz communications. The designed waveform exploits $99.3 \%$ of the total in-band energy admissible by this mask and provides an extra degree of freedom for out-of-band interference management. A proof-of-concept experiment is conducted using a $\mathbf{3 0 0}$ GHz photonics-based terahertz communications link to demonstrate the generation and wireless transmission of the proposed waveform. Experimentally validated bit error rates show that the proposed waveform outperforms the widely adopted raisedcosine waveform and the better-than-Nyquist waveform even when the Nyquist criterion for inter-symbol interference-free signaling is not satisfied. Moreover, an error-free transmission at $1.44 \mathrm{Gbit} / \mathrm{s}$ is achieved without employing complicated digital signal processing at the receiver side.
\end{abstract}

Index Terms-IEEE 802.15.3d-2017 Standard, pulse shaping, spectral radiation efficiency (SRE), terahertz communications, terahertz photonics, waveform design.

\section{INTRODUCTION}

C ONTINUOUSLY increasing demands for short-range high-speed wireless communications have stimulated intensive research interests in innovative solutions towards spectrally efficient communications technologies amid the radio spectrum, which is fully allocated up to $100 \mathrm{GHz}$. As an unoccupied part in the electromagnetic spectrum, the terahertz band from $100 \mathrm{GHz}$ to $10 \mathrm{THz}$ has emerged as a promising

Mohamed Shehata and Withawat Withayachumnankul are with the Terahertz Engineering Laboratory, School of Electrical and Electronics Engineering (EEE), Faculty of Engineering, Computer and Mathematical Sciences (ECMS), The University of Adelaide, Adelaide, 5005 SA e-mail: (mohamed.shehata@adelaide.edu.au; withawat@adelaide.edu.au).

Ke Wang is with the School of Engineering, RMIT University, Melbourne, Victoria, Australia. e-mail: (ke.wang@rmit.edu.au).

Julian Webber, Masayuki Fujita, and Tadao Nagatsuma are with the Graduate School of Engineering Science, Osaka University, Osaka, Japan. e-mail:(nagatsuma@ee.es.osaka-u.ac.jp; fujita@ee.es.osaka-u.ac.jp; webber@ee.es.osaka-u.ac.jp).

Manuscript received Month XX, 2020; revised Month XX, 2020; accepted Month XX, 2020. Date of publication Month XX, 2020; date of current version Month XX, 2020. The authors wish to acknowledge support from the Australian Research Council Discovery Project (No. ARC DP180103561), The Australian Government through the Australian Government Research Training Program Scholarship, The University of Adelaide through the Adelaide Scholarship International, and the Core Research for Evolutional Science \& Technology (CREST) program of the Japan Science and Technology Agency (JST) (JPMJCR1534). solution to meet these demands [1]. To accommodate multiuser access to this band, the maximum effective isotropic radiated powers (EIRPs) of practical wireless transmitters and their usable bandwidths are regulated by the local regulatory authorities through the globally-adopted wireless communication standards. To meet the spectral emission requirements of these standards, the over-the-air (OTA) signals' spectra are reshaped at the transmitter side before being wirelessly distributed over free-space channels [2], [3]-[9].

In the field of terahertz communications, the first worldwide standard, officially called the IEEE 802.15.3d Standard [10], was released in 2018 to support the operation of high-speed Ethernet wireless links up to $100 \mathrm{Gbit} / \mathrm{s}$. In this standard, a $69 \mathrm{GHz}$ bandwidth is dedicated to terahertz communications in the frequency range from $252 \mathrm{GHz}$ to $321 \mathrm{GHz}$, commonly called the IEEE terahertz band. It is important to highlight that, although the IEEE 802.15.3d Standard does not specify a pulse shaping filter, filtering is implied in the spectral emission masks specified by this standard. Despite the broad bandwidth allocated by the IEEE 802.15.3d Standard to terahertz communications, the power levels offered by currently available terahertz wireless transmitters are relatively limited [11], [12]. The power is further reduced at the receiver side due to the high free-space path loss at terahertz frequencies. Hence, the prudent utilization of the emission masks admissible by the IEEE 802.15.3d Standard raises a key challenge in the development of future terahertz communications systems. Despite its importance, this challenge remains under-investigated.

In most of the earlier demonstrations, the terahertz spectrum has been explored freely without imposing any constraints on the EIRPs, the central frequencies and the bandwidths of the generated terahertz signals [13]-[17]. Only a limited number of these demonstrations have partially considered the physical (PHY) layer specifications defined by the IEEE 802.15.3d Standard [10], including the pulse shaping required to comply with this standard. For instance, in [12], [18], the first successful transmission of an IEEE 802.15.3d terahertz signal in the $300 \mathrm{GHz}$ band was reported. A raised cosine (RC) filter, with a roll-off factor of 0.35, was employed to reshape the spectrum of a quadrature amplitude modulated (QAM) signal in the baseband before being up-converted and transmitted over the IEEE band at data rates of 56 , 80 and $100 \mathrm{Gbit} / \mathrm{s}$. In [19], an all-electronic transmitter was implemented to demonstrate the generation of a $27 \mathrm{GHz}$ bandwidth signal at $300 \mathrm{GHz}$. The generated terahertz signal spanned 12.5 single-user channels of $2.16 \mathrm{GHz}$ bandwidth 
each, which violates the frequency allocation plan defined by the IEEE 802.15.3d Standard for multi-user operation schemes [10]. Moreover, the design criteria of the generated signal spectrum were not reported. In [20], a dual-channel generation and transmission of $2.16 \mathrm{GHz}$ bandwidth terahertz signals in the $300 \mathrm{GHz}$ band was reported. An RC pulse shaping filter, with a roll-off factor of 0.35 , was employed to reshape the signals spectra in the baseband. The conformity of the generated spectra to the emission mask defined in [10] was not investigated either. Obviously, a common shortcoming in the aforementioned demonstrations is the lack of compliance to this IEEE Standard. As a preliminary investigation, in [21] we proposed the first IEEE 802.15.3d Standard-compliant waveform.

To further address this issue, here we provide an analytical framework for the design and performance assessment of terahertz waveforms developed for IEEE 802.15.3d terahertz communications. Then, we investigate two widelyused waveforms, namely the RC and the better-than-Nyquist (BTN) waveforms, using the developed analytical framework. Importantly, we propose an alternative waveform for terahertz communications under the most stringent spectral constraints defined by the IEEE 802.16.3d Standard. The transmission performance of the three considered waveforms is experimentally investigated using a photonics-based terahertz communication system, with the bit error rate (BER) as a performance metric. It should be highlighted that, using photonics-based terahertz systems for experimental demonstrations emphasizes the possibility of seamless integration of radio-over-fiber passiveoptical-networks (RoF-PONs) to terahertz wireless links as well as the applicability of microwave-photonic (MWP) signal processing techniques, at least conceptually, to terahertz communications [13].

The rest of this paper is organized as follows. Section II overviews the IEEE 802.15.3d Standard and introduces a relevant analytical framework for the terahertz waveform design. In Section III, two of the most commonly used pulse shapes in digital communications are analyzed and the proposed waveform is presented. Section IV presents the experimental investigation of the transmission performance of the three considered waveforms using a terahertz photonic system. The paper is concluded in Section V.

\section{Problem Formulation and Design Guidelines}

Since the IEEE 802.15.3d Standard-compliant terahertz waveforms are subject to a set of spectral constraints, designing the targeted waveform in the frequency domain followed by frequency-to-time conversion is a straightforward approach. However, reshaping the spectra of narrow band signals in the terahertz band is not practical when using the currently available all-electronic digital platforms. Therefore, to relax the computational efforts required, we map the IEEE spectral emission mask from the passband to the baseband [22]. Then, the baseband spectrum is reshaped and up-converted to the passband to produce the spectrally compliant terahertz OTA signal. This approach facilitates the efficient utilization of the all-electronic-based processing platform.
Since the IEEE 802.15.3d Standard relies on multi-rate adaptive coding and modulation (ACM) schemes, the proposed design approach can be considered highly adaptive as it allows for a modular transmitter design and implementation using all-electronic software-defined radio (SDR) platforms based on field-programmable gate arrays (FPGAs), regardless of the terahertz carrier frequency and/or the generation technique. These platforms are generic as they have the capacity to implement sophisticated communications processing in the baseband, including the pulse shaping and the ACM [23]. The implementable modulation schemes can be simple onoff keying (OOK) and binary phase shift keying (BPSK), or higher-order vector modulation formats such as the $\pi / 2$ quadrature phase shift keying ( $\pi / 2$-QPSK), 16-quadrature amplitude modulation (16-QAM) and 64-QAM. Moreover, the transmission bit rates supported by all-electronic SDR-driven optical systems can exceed $100 \mathrm{Gbit} / \mathrm{s}$ [24]. It is noteworthy that all of the aforementioned modulation formats and data rates are compatible with the technical requirements defined in the IEEE 802.15.3d Standard [10].

\section{A. The IEEE 802.15.3d Standard Spectral Emission Mask}

The IEEE 802.15.3d Standard defines eight different channelization schemes within the range form $252 \mathrm{GHz}$ to $321 \mathrm{GHz}$. Each channelization scheme comprises multiple parallel channels with bandwidths ranging from $2.16 \mathrm{GHz}$ to $69.1 \mathrm{GHz}$. To limit the mutual inter-band interference (IBI) among the OTA terahertz signals transmitted in adjacent channels, a spectral emission mask is imposed to the EIRP of each signal. The spectral emission mask of the $i^{\text {th }}$ channelization scheme, denoted by $M_{i}(f)$, can be modeled by a piecewise linear function when expressed in the $\mathrm{dBr}$ unit. This spectral mask is defined by a unique set of four knee frequencies, denoted by $f_{i, k}$, and the corresponding power spectral density (PSD) upper-bounds at these frequencies. Here, $i \in\{1,2 \ldots, 8\}$ denotes the channelization scheme number and $k \in\{0,1,2,3,4\}$ is the knee frequency index. This can be formulated mathematically as follows:

$M_{i}(f)(\mathrm{dBr})=$

$$
\begin{cases}\left(\frac{M_{i, k}-M_{i, k-1}}{f_{i, k}-f_{i, k-1}}\right) f-\left(\frac{M_{i, k+1}-M_{i, k}}{f_{i, k+1}-f_{i, k}}\right) f_{i, k}+M_{i, k} \\ & ; f_{i, k-1} \leq f<f_{i, k} \\ \leq-30 & ; f \geq f_{i, 4} .\end{cases}
$$

Tables I and II list the numerical values of the knee frequencies and the corresponding PSD limits for the eight channelization schemes specified by the IEEE 802.15.3d Standard. It should be pointed out that the standard does not specify the absolute EIRP level as it is dependent on the respective local regulating authorities.

\section{B. Spectral Compliance Metric}

Several metrics have been introduced to quantify the compliance of a given signal spectrum with a pre-specified spectral emission mask [25], [26]. In [27], a frequency-dependent 
TABLE I

KNEE FREQUENCIES OF THE SPECTRAL EMISSION MASKS OF THE IEEE 802.15.3D STANDARD [10]. FREQUENCIES ARE IN GHZ

\begin{tabular}{cccccc}
\hline ID & BW & $f_{i, 1}$ & $f_{i, 2}$ & $f_{i, 3}$ & $f_{i, 4}$ \\
\hline 1 & 2.16 & 0.94 & 1.1 & 1.6 & 2.20 \\
2 & 4.32 & 2.02 & 2.18 & 2.68 & 3.28 \\
3 & 8.64 & 4.18 & 4.34 & 4.84 & 5.44 \\
4 & 12.96 & 6.34 & 6.50 & 7.00 & 7.60 \\
5 & 17.28 & 8.50 & 8.66 & 9.16 & 9.76 \\
6 & 25.92 & 12.82 & 12.98 & 13.48 & 14.08 \\
7 & 51.84 & 25.78 & 25.94 & 26.44 & 27.04 \\
8 & 69.12 & 34.42 & 34.58 & 35.08 & 35.68 \\
\hline
\end{tabular}

TABLE II

PSD LIMITS OF THE SPECTRAL EMISSION MASKS OF THE IEEE 802.15.3D STANDARD [10]. $M_{i, k}$ IS IN dBr UNIT.

\begin{tabular}{ccccc}
\hline$M_{i, o}$ & $M_{i, 1}$ & $M_{i, 2}$ & $M_{i, 3}$ & $M_{i, 4}$ \\
\hline 0 & 0 & -20 & -25 & -30 \\
\hline
\end{tabular}

binary compliance metric, denoted by $B_{\tilde{\Psi}}(f) \in\{0,1\}$, has been proposed to quantify the compliance of ultra-wideband (UWB) signals' spectra with the FCC spectral emission mask. Likewise, this metric can be employed in the context of IEEE 802.15.3d Standard-compliant terahertz communications. For an arbitrary terahertz signal envelope $\psi(t)$ with a normalized $\operatorname{PSD}$ of $\left|\tilde{\Psi}_{n}(j f)\right|^{2}, B_{\tilde{\Psi}}(f)=1$ if $\left|\tilde{\Psi}_{n}(j f)\right|^{2}$ does not exceed $M_{i}(f)$ at $f$, and $B_{\tilde{\Psi}}(f)=0$ if these spectral constraints are exceeded. This point-wise definition can be reformulated mathematically as follows:

$$
B_{\tilde{\Psi}}(f)=\frac{1}{2}(\mathrm{U}(\zeta)-\mathrm{U}(-\zeta)+1),
$$

where $\zeta(f)=10 \log \left(M_{i}(f) /\left|\tilde{\Psi}_{n}(j f)\right|^{2}\right)$, and $\mathrm{U}(\zeta)$ is the Heaviside unit step function, defined as $\mathrm{U}(\zeta)=1$ if $\zeta \geq 0$ and $\mathrm{U}(\zeta)=0$ if $\zeta<0$. It should be pointed out that, in contrast to the definition provided in [27], $\zeta(f)$ is defined in the $\mathrm{dBr}$ unit, rather than in the linear unit, to capture spectral details at low PSD levels such as the additive white Gaussian noise (AWGN) floor level. Hence, this modified metric can precisely identify the frequencies at which the signal PSD should be particularly considered. Meanwhile, it is also reasonable to define a figureof-merit (FoM) that quantifies the overall compliance of the signal PSD over an arbitrary bandwidth of interest. We define the average compliance coefficient $(\mathrm{CC})$, denoted by $\rho_{\tilde{\Psi}}$, as:

$$
\rho_{\tilde{\Psi}}=\frac{1}{2 \Delta f} \int_{f_{0}-\Delta f}^{f_{0}+\Delta f} B_{\tilde{\Psi}}(f) d f,
$$

where $0 \leq \rho_{\tilde{\Psi}} \leq 1, f_{0}$ is the central terahertz carrier frequency of interest, and $2 \Delta f$ is the bandwidth over which the CC of $\left|\tilde{\Psi}_{n}(j f)\right|^{2}$ is calculated. Although the IEEE 802.15.3d Standard [10] limits the operation of a terahertz signal to the passband bandwidths listed in Table $\mathrm{I}$, the value of $\Delta f$ in (3) can be arbitrarily large $(\Delta f \gg \mathrm{BW})$ to capture the aggregate contributions of the spurious out-of-band (OOB) emissions and the spectral regrowth effects in the overall compliance analysis.

\section{Spectral Radiation Efficiency}

The concept of maximizing the spectral radiation efficiency (SRE) of an emitted OTA signal, whilst complying with the constraints of a spectral emission mask, has been well-known in the context of UWB communications [27]. In this work, we apply this concept to the design of IEEE 802.15.3d-compliant signals. Assume that $\left|\tilde{\Psi}_{n}(j f)\right|^{2}$ is defined over the bandwidth of an IEEE 802.15.3d channel. The SRE of this envelope signal is defined as the ratio of the power contained within its spectrum to the total PSD admissible by the spectral emission mask. This definition is expressed mathematically as follows:

$$
\eta=\frac{\int_{\mathrm{BW}}\left|\tilde{\Psi}_{n}(j f)\right|^{2} d f}{\int_{\mathrm{BW}} M_{i}(f) d f},
$$

where $\eta$ is the SRE of the baseband envelope of the terahertz signal.

\section{Problem Formulation}

A number of benefits can be achieved by maximizing the SRE of terahertz signals at the receiver front-end, including the improvement of the signal-to-noise plus interference ratio (SNIR) and extending the wireless communications reach. An implicit challenge arises when considering (3) and (4) jointly, where the PSD of an optimal terahertz waveform should be carefully shaped to achieve the maximum SRE, and at the same time, be subject to the EIRP constraints imposed by the IEEE 802.15.3d spectral regulation mask. This design tradeoff leads to the definition of the following non-convex multiobjective optimization problem:

Problem Statement. An optimal terahertz signal that fully complies with the spectral emission mask of the IEEE 802.15.3d Standard can be obtained by designing its baseband envelop waveform $\psi(t)$, defined by a complete parameter set of $\Xi$, such that the following two independent optimization problems are jointly satisfied:

$$
\underset{\Xi}{\operatorname{maximize}}\left(\int_{\mathrm{BW}}\left|\tilde{\Psi}_{n}(j f, \Xi)\right|^{2} d f\right)
$$

such that

$$
\max \left\{\left|\tilde{\Psi}_{n}(j f, \Xi)\right|^{2}\right\} \leq M(f) \forall f \text { or } \rho_{\tilde{\Psi}}=1 .
$$

The solution of the multi-objective optimization problem defined in (5) and (6) jointly yields a desired optimal terahertz waveform.

\section{TerahertZ WAVEFORMS FOR THE IEEE 802.15.3D STANDARD}

\section{A. Conventional Signaling Waveforms}

The RC pulse shape has been widely adopted for signaling over the physical layers of various wireless and optical communications systems. However, in non-terahertz wireless communications, several reports have demonstrated pulse shape 
designs that can outperform, or at least be comparable with, the RC pulse. In [28] it has been proven that, compared with the RC waveform, employing the BTN waveform in the generalized frequency division multiplexing (GFDM) systems can potentially improve the symbol error rate (SER) performance. In [29], the transmission performance of the BTN waveform has been experimentally tested using a visible light communication system operating under AWGN channel conditions. It was found that the BTN pulse shape offers superior bit-error rate (BER) performance and lower computational complexity compared with the RC pulse shape.

In this work, we adopt the RC and the BTN waveforms as comparison benchmarks. The spectrum of an $\mathrm{RC}$ waveform is given by [30]

$$
\begin{aligned}
& S_{\mathrm{RC}}(f)= \\
& \begin{cases}1, & 0 \leq|f|<B(1-\alpha) \\
\frac{1}{2}\left\{1+\cos \left(\frac{\pi}{2 B \alpha}(|f|-B(1-\alpha))\right)\right\}, & B(1-\alpha) \leq|f| \\
& \leq B(1+\alpha) \\
0, & B(1+\alpha) \leq|f|,\end{cases}
\end{aligned}
$$

where $T_{s}=1 / R_{s}$ is the symbol duration, $R_{s}$ is the symbol rate, $\alpha$ is the roll-off factor and $B=1 /\left(2 T_{s}\right)$ is the minimum transmission bandwidth. The corresponding RC time-domain waveform is given by [30]

$$
s_{\mathrm{RC}}(t)=\operatorname{sinc}\left(\frac{t}{T_{s}}\right) \frac{\cos \left(2 \pi \alpha t / T_{s}\right)}{1-4 \alpha^{2} t^{2} / T_{s}^{2}} .
$$

The spectrum of the BTN pulse shape is defined as follows [30]:

$$
\begin{aligned}
& S_{\mathrm{BTN}}(f)= \\
& \begin{cases}1, & 0 \leq|f|<B(1-\alpha) \\
\exp \left\{\frac{\ln 2}{\alpha B}|f-B(1-\alpha)|\right\}, & B(1-\alpha) \leq|f|<B \\
1-\exp \left\{\frac{\ln 2}{\alpha B}|B(1+\alpha)-f|\right\}, & B \leq|f|<B(1+\alpha) \\
0, & B(1+\alpha) \leq|f| .\end{cases}
\end{aligned}
$$

The corresponding time-domain BTN waveform is given by [30]

$$
\begin{aligned}
& s_{\mathrm{BTN}}(t)= \\
& 2 B \operatorname{sinc}(2 B t) \times \frac{4 \beta \pi t \sin (2 \pi B \alpha t)+2 \beta^{2} \cos (2 \pi B \alpha t)-\beta^{2}}{4 \pi^{2} t^{2}+\beta^{2}},
\end{aligned}
$$

where $\beta=(\ln 2 / \alpha B)$. To satisfy the IEEE $802.15 .3 \mathrm{~d}$ Standard, the value of $T_{s}$ in (7)-(10) is selected according to the discrete set of the symbol rates defined in [10]. Therefore, $\alpha$ is the only parameter that can be tuned to control the PSDs of the RC and the BTN waveforms. It should be pointed out that, practically, the RC and the BTN pulse shaping are divided between the transmitter and the receiver sides. Each stage employs the square root of (7) and (9).

\section{B. Proposed Waveform: Frequency and Time Domain Analysis}

Despite their unique temporal and spectral features, Lorentzian-based pulse shapes have been overlooked in the context of spectral shaping for digital communications [31]. An amplitude-scaled Lorentzian pulse can be expressed as [31]:

$$
p_{L}(t)=\frac{A}{1+\left(\frac{t}{\tau}\right)^{2}},
$$

whereas the Fourier transform of $p_{L}(t)$ is given by [31]

$$
L(f, \tau)=A \pi \tau \exp (-2 \pi \tau|f|) .
$$

When expressed in the $\mathrm{dBr}$ unit, $L(f, \tau)$ follows a triangularshaped spectrum with a steady spectral roll-off [32], which can be controlled via the temporal width $\tau$ of the Lorentzian basis pulse. This spectral feature makes it possible to use the Lorentzian pulse to match the spectral mask shape defined in (1). In particular, we propose a fully-compliant spectrum based on a linear combination of logarithmic double-sided Lorentzian spectra centered at the knee frequencies as follows:

$$
\begin{aligned}
\mathcal{L}(f) \quad(\mathrm{dB}) & =\sum_{k=1}^{4} A_{k}\left(L_{k}^{+}\left(f, \tau_{k}\right)+L_{k}^{-}\left(f, \tau_{k}\right)\right) \\
& =-\sum_{k=1}^{4}\left(b_{k}\left(\left|f+f_{k}\right|+\left|f-f_{k}\right|\right)+2 a_{k}\right),
\end{aligned}
$$

where $L_{k}^{ \pm}\left(f, \tau_{k}\right)=10 \log \left(L\left(f \pm f_{k}, \tau_{k}\right)\right), b_{k}=10 \tau_{k} \log (e)$ and $a_{k}=10 \tau_{k} \log \left(\pi A_{k} \tau_{k}\right)$. Equation (13) suggests that the area under $M_{i}(f)$ can be fully occupied by four overlapping pairs of the triangular logarithmic Lorentzian spectra, corresponding to the four knee frequencies defined in (1).

To reduce the mathematical complexity associated with applying the inverse Fourier transform directly to the linear form of (13), we inspect the distribution of the emission mask PSD over frequency. The total power contained in a sub-band located between two consecutive knee frequencies is given by:

$$
P_{k}=\frac{\int_{f_{k-1}}^{f_{k}} M_{i}(f) d f}{\int_{0}^{f_{4}} M_{i}(f) d f} ; k \in\{1,2,3,4\},
$$

where $f_{0}=0$. Accordingly, the cumulative PSD contribution of $M_{i}(f)$ at the $k^{\text {th }}$ knee frequency can be defined as follows:

$$
C_{k}=\sum_{m=1}^{k} P_{m} ; k \in\{1,2,3,4\} .
$$

Figure 1 illustrates $P_{k}$ and $C_{k}$ versus $k$ for the first channelization scheme specified in [10]. From this figure, it is observed that, more than $95 \%$ of the PSD under the spectral emission mask is concentrated in the frequency range of $-f_{2} \leq f \leq f_{2}$. This property holds for the eight IEEE 802.15.3d channelization schemes and can be utilized to simplify (13) to the following form:

$$
\begin{aligned}
\mathcal{L}(f)(\mathrm{dB}) & \approx L_{1}^{+}\left(f, \tau_{1}\right)+L_{1}^{-}\left(f, \tau_{1}\right) \\
& =2 a_{1}-b_{1}\left(\left|f+f_{1}\right|+\left|f-f_{1}\right|\right) .
\end{aligned}
$$


The corresponding PSD, measured in the units of $\mathrm{W} / \mathrm{Hz}$, is given by:

$$
|\mathcal{L}(f)|^{2}=\exp (2 \gamma B \bar{\alpha}) \exp (-\gamma(|f+B \bar{\alpha}|+|f-B \bar{\alpha}|)),
$$

where $\gamma=\pi \tau$, and $\bar{\alpha}=1-\alpha$, which is defined as the complementary roll-off factor.

The time-domain waveform corresponding to (17) can be obtained by applying the inverse Fourier transform to the square root of (17), which yields:

$$
\begin{aligned}
s_{\mathcal{L}}(t) & \frac{1}{2 \pi} \int_{-\infty}^{+\infty} \sqrt{\mathcal{L}(f)} \exp (j \omega t) d \omega \\
= & \exp (\gamma B \bar{\alpha}) \pi \exp \left(-\gamma \frac{\bar{\alpha}}{T_{s}}\right) \\
& \times\left\{\frac{\bar{\alpha}}{T_{s}} \operatorname{sinc}\left(\frac{\bar{\alpha} t}{T_{s}}\right)+\frac{1}{\gamma} \frac{\cos \left(\frac{\pi \bar{\alpha} t}{T_{s}}\right)-\left(\frac{\pi t}{\gamma}\right) \sin \left(\frac{\pi \bar{\alpha} t}{T_{s}}\right)}{1+\left(\frac{\pi t}{\gamma}\right)^{2}}\right\} .
\end{aligned}
$$

It is noted from (18) that the Nyquist sampling conditions for inter-symbol interference (ISI)-free signaling is not strictly satisfied by $s_{\mathcal{L}}(t)$, i.e., $s_{\mathcal{L}}(t=0)=1$, whereas $s_{\mathcal{L}}\left(t=n T_{s}\right) \neq 0$ when $n=\{ \pm 1, \pm 2, \ldots\}$.

Alternatively, the ISI performance of $s_{\mathcal{L}}(t)$ can be evaluated based on the relaxed Nyquist condition as follows [33]:

$$
\text { ISI }(\mathrm{dB})=10 \log \frac{\left|s_{\mathcal{L}}(0)\right|^{2}}{\left|\sum_{n=-\infty ; n \neq 0}^{+\infty} s_{\mathcal{L}}\left(t-n T_{s}\right)\right|^{2}} .
$$

This condition considers a signaling waveform to be ISI-free as long as the instantaneous signal-to-ISI power ratio at the optimum sampling time instant is maintained above a prespecified acceptable threshold. In Section D, the condition in (19) is employed to quantify the ISI performance of the proposed waveform after optimizing its spectrum.

\section{Comparison of the Waveform' Spectra}

Figure 2 compares the impact of reshaping the spectra of the three considered waveforms on their compatibility to the IEEE 802.15.3d spectral requirements. Specifically, Figs. 2(a) and 2(b) show the RC and the BTN spectra at different values of $\alpha$, whereas Fig. 2(c) plots the proposed spectrum at $\alpha=0$ and different values of $\gamma$. It noteworthy that, for the RC and the BTN waveforms, the spectral flatness and the IBI are jointly controlled with only a single degree of freedom, which is $\alpha$, whereas, according to (17), the proposed spectrum possesses two independent degrees of freedom to manage the spectral flatness and the IBI, namely, $\alpha$ and $\gamma$. These properties make it possible to manage the SRE and the compliance independently and more flexibly using the proposed spectrum than with the RC and BTN waveforms. Additionally, in the proposed waveform, the impact of the complementary roll-off

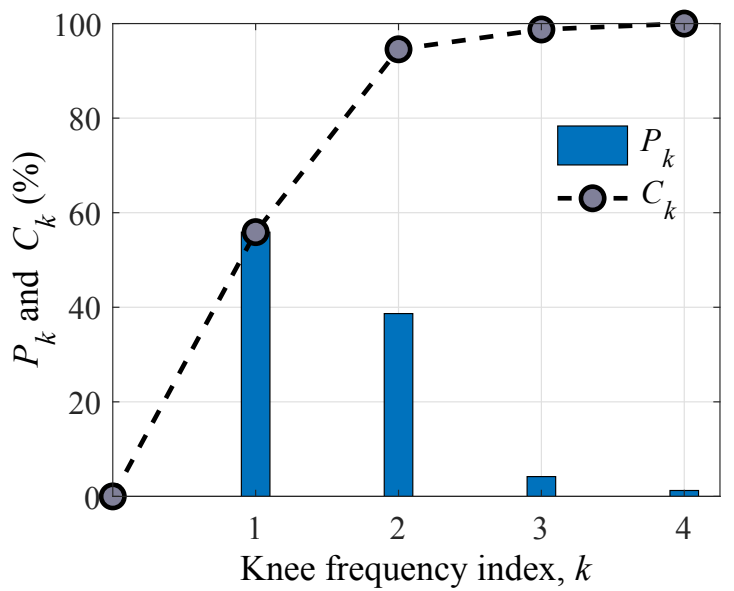

Fig. 1. Distribution of the PSD over the sub-bands of spectral emission mask defined by the IEEE 802.15.3d Standard for the first channelization scheme [10]. $P_{k}$ is for power distribution in each sub-band, whereas $C_{k}$ is for cumulative power. The dashed line is for visual guidance.

factor, i.e., $\bar{\alpha}$, is the same as the impact of $\alpha$ in the $\mathrm{RC}$ and the BTN waveforms; increasing both parameters increase the transmission bandwidth.

\section{Waveform Design Optimization}

We optimize the design of each waveform to fit the spectral emission mask defined by the IEEE 802.15.3d Standard [10] for the first channelization scheme, which has the most stringent emission mask with a steady roll-off as large as $20 \mathrm{~dB}$ over a transition bandwidth of only $160 \mathrm{MHz}$. Although the waveforms considered in this work are optimized to fit the first channelization spectral mask, the conducted analysis is general and also applicable to other channelization schemes. This is because the bandwidths of all channelization schemes are integer multiples of $2.16 \mathrm{GHz}$ as shown in Table I.

To ensure that the designed spectrum is compliant with the first emission mask, the involved parameters are optimized for each waveform individually, based on (5) and (6). Moreover, we assume a maximum frequency of $32 \mathrm{GHz}$ to define the spectra of the three spectral shapes. This maximum bandwidth is large enough compared to the baseband equivalent bandwidth of the considered channelization scheme $(1.08 \mathrm{GHz})$ and, consequently, guarantees aliasing-free spectra. For the three spectral shapes, the value of $\alpha$ is changed from 0 to 1 with a step of $10^{-3}$. For the RC and the BTN waveforms, $B$ is set to $R_{\mathrm{s}} / 2$, where $R_{\mathrm{s}}=1.44 \mathrm{Gbit} / \mathrm{s}$, while for the proposed waveform, $B$ is set to $f_{0}$. Moreover, for the proposed spectrum, since $\gamma$ relies on the full width at half maximum (FWHM) $\tau$, the value of $\tau$ is increased from 0 to $20 T_{s}$ with a resolution of $0.001 T_{s}$. The SRE and CC are evaluated for the three pulse shapes over the considered parameters ranges. Then, the direct search algorithm (DSA) [27] is applied to the calculated SRE and CC to extract the optimum values of $\alpha$ and $\gamma$.

Figure 3(a) shows the SRE and the CC versus $\alpha$ for the $\mathrm{RC}$ and the BTN waveforms, whereas Fig. 3(b) shows the SRE and the CC versus $\gamma$ for the proposed waveforms at the optimized value of $\alpha$. From Fig. 3(a), it can be seen that 

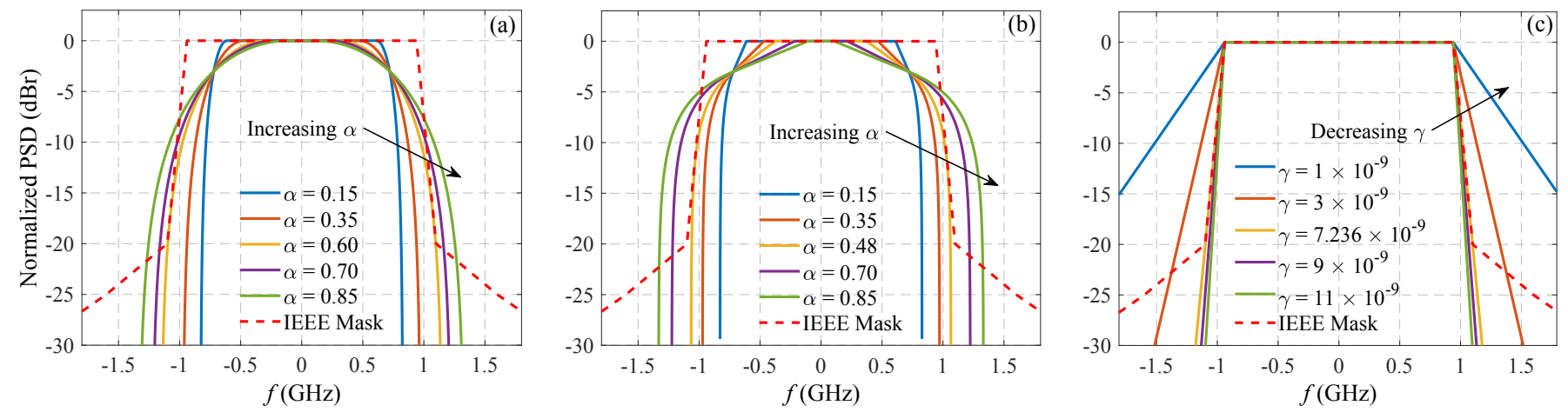

Fig. 2. Simulated baseband spectra of the (a) RC waveform, (b) the BTN waveform, and (c) the proposed waveform. The roll-off factor is fixed at $\alpha=0$ for the proposed waveform at all value of $\gamma$.
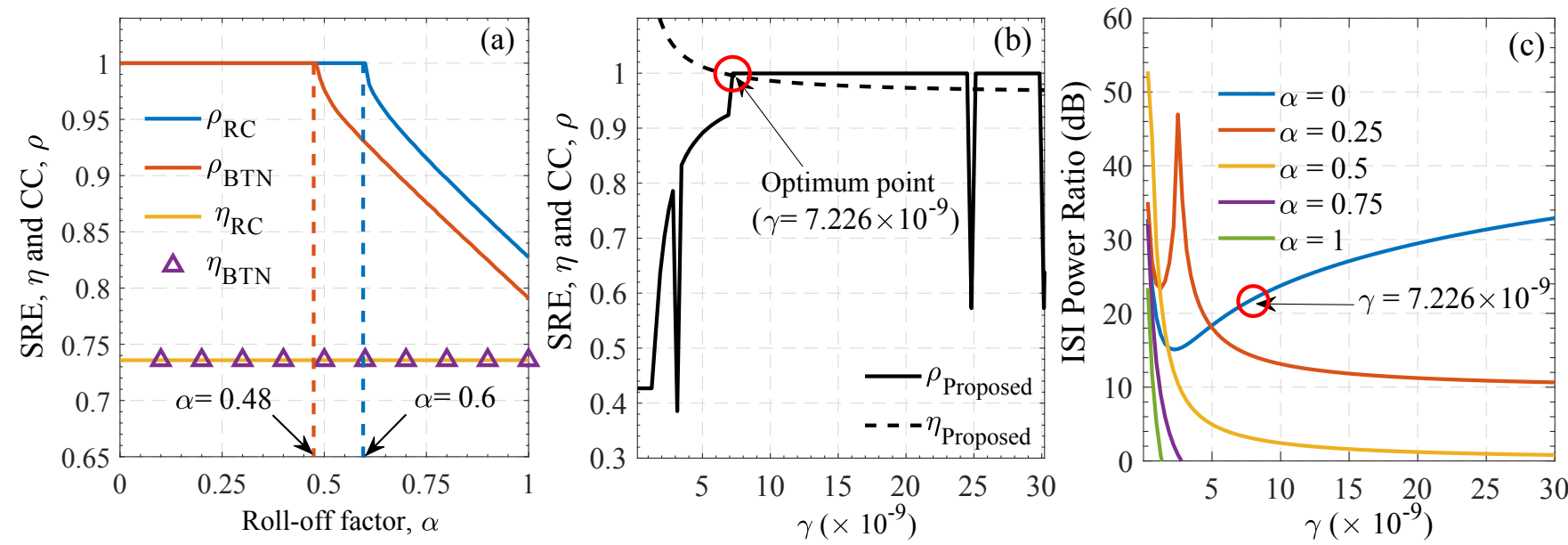

Fig. 3. Design optimization metrics of the three considered waveforms. (a) the compliance and spectral radiation efficiency versus $\alpha$ for the RC and BTN waveforms, (b) the compliance and spectral radiation efficiency versus $\gamma$ for the proposed waveform at a $\alpha=0$, and (c) the ISI performance versus $\gamma$ for the proposed waveform at different values of $\alpha$.

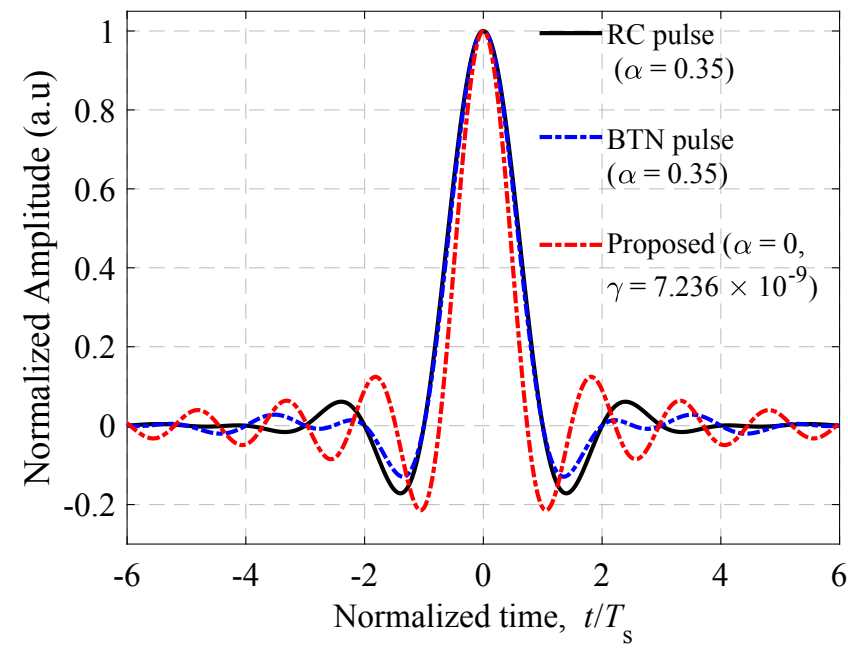

Fig. 4. Three waveforms obtained from the optimal parameters to comply with the IEEE-defined spectral emission mask with the highest SRE.

the RC and the BTN waveforms fully comply with the IEEE 802.15.3d emission mask constraints with a roll-off factor $\alpha$ up to 0.48 and 0.6 , respectively. Beyond these values, the compliance condition is violated, which is strictly prohibited for IBI considerations in multi-user channel access scenarios. The SRE is approximately independent of $\alpha$ with a constant value of $73.58 \%$ for both waveforms. As can be observed from Figs. 2(a) and 2(b), this independence is a result of substituting the unfilled parts under the emission mask at $|f|<B$ by the spectrally broadening of the PSD for $|f|>B$ and hence; maintaining a constant total power. Therefore, in this work, the roll-off factor is selected at the commonly used value of $\alpha=0.35$ to define the optimum RC and the BTN waveforms.

For the proposed waveform, the full compliance is achieved at $\alpha=0$ and a spectral roll-off control factor, $\gamma$, of $7.226 \times$ $10^{-9}$, whereas the corresponding SRE is $99.3 \%$ as can be seen from Fig. 3(b). The dips observed in the CC are due to the adopted Heaviside step function in the definition of this metric as well as the non-differentiability of the spectral emission mask at the knee frequencies. This difference between the SRE of the proposed waveform and that of the RC and the BTN waveforms implies a virtual increase of about $34.96 \%$ in the emitted terahertz power at no additional cost. It is noteworthy that, in [11], a $20 \%$ increase in the emitted terahertz power was achieved via the monolithic integration of two photonic transmitters. This comparison emphasizes the importance of spectral shaping in managing the available power budget.

Since the proposed waveform is not ISI-free, the ISI per- 

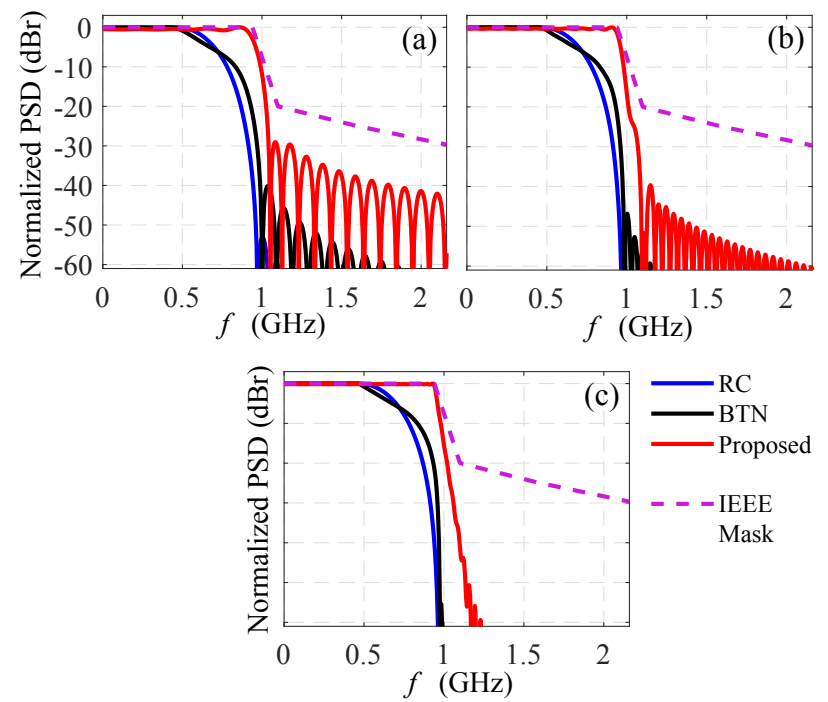

Fig. 5. Impact of hard truncation on the stop-band attenuation of the RC, BTN and the proposed waveforms, (a) $L=7$, (b) $L=15$, and (c) $L=41$. The dashed lines represent maximum OOB emission limit allowed by the IEEE 802.15.3d [10].

formance of this waveform is evaluated using a $2^{10}$ randomly generated BPSK symbols [30], which interfere a pulse centered at $t=0$ with no AWGN. Figure 3(c) shows the result of ISI based on (19) of the proposed waveform versus $\gamma$ at different values of $\alpha$. At the optimum values of $\alpha$ and $\gamma$, the proposed waveforms experiences a signal-to-ISI power ratio of $21.93 \mathrm{~dB}$, which is high enough to ignore its impact during the matched filtering at the receiver side [33]. Figure 4 shows the three considered waveforms resulting from the optimization of their respective parameters $\alpha$ and $\gamma$.

In practice, the pulse shaping process is implemented in the digital domain using finite impulse response filter (FIRs). Here, a truncated version of the signaling waveform is generated with an adequately finite filter length, denoted by $L$, to minimize the group delay of the FIR filter. However, this truncation induces undesired side-lobe level (SLL) spectral components beyond the dedicated waveform bandwidth. For an RC waveform with a symbol span of $L=5$ and a roll-off factor of 0.35 , this SLL can be as high $-1.5 \mathrm{~dB}$, which strongly violates the maximum stop-band attenuation of $-30 \mathrm{~dB}$ [33]. Figures 5(a)-(c) shows the impact of hard truncation on the stop-band attenuation of the three considered waveforms for a symbol span of $2 L$, where $L=7,15$ and 41 , respectively. For the proposed waveform, an almost flat passband response is exhibited up to the first knee frequency, i.e., $f_{1}=0.94 \mathrm{GHz}$, while offering a maximum SLL of about $-30 \mathrm{~dB}$. Moreover, although the proposed waveform shows SLL that is higher than the RC and the BTN waveforms, this SLL is still lower than the $-30 \mathrm{dBr}$ PSD limit defined in (1) for OOB emissions. By using a sufficiently long symbol span, i.e., $L>41$, the SLL can be maintained well below this limit and the spectrum can be fit to the emission mask more efficiently.

\section{EXPERIMENTS AND RESULTS}

As a proof-of-concept, the transmission performance of the three considered waveforms is experimentally evaluated using the photonic-terahertz communications system shown in Fig. 6. This system is based on the principles of intensity modulation-direct detection (IM-DD) and photo-mixing. In the experiment, the spectrum of a $2^{20}-1$ pseudo-random binary sequence is reshaped by each of the three considered pulse shapes to generate the baseband signals. A regular pattern of consecutive of "1"s and "0"s, running at a symbol rate of $R_{s} / 4$, is employed as a synchronization word. This synchronization word is interleaved cyclically with the data packets. A guard time interval of 100 zero-valued symbols is inserted between consecutive data packets.

This baseband signal is generated using a 64 GSample/s arbitrary waveform generator (AWG) with a voltage of $V_{p}=300$ $\mathrm{mV}$ and a data rate of $1.44 \mathrm{Gbit} / \mathrm{s}$. This rate is compatible with the first channelization scheme specified in [10]. Two optical carriers at $193.5000 \mathrm{THz}$ and $193.8100 \mathrm{THz}$ are emitted by a dual-channel continuous wave tunable laser source (CWTLS) and combined by a 50:50 optical coupler (OC). The linewidth of this CW-TLS is about $100 \mathrm{kHz}$. The generated baseband signal is then modulated onto the combined optical carriers by a Mach-Zhender modulator operated in the linear region of its electro-optic transfer characteristics. After optical amplification, the two optical signals are injected into a UTCPD for photo-mixing. The terahertz carrier frequency is 311.00 $\mathrm{GHz}$, which corresponds to the $32^{\text {nd }}$ channel defined by the Standard. In the experiment, the photo-current is increased from $1 \mathrm{~mA}$ to $6 \mathrm{~mA}$, with a step of $1 \mathrm{~mA}$. Table III lists the UTC-PD photo-current, denoted by $I_{\mathrm{ph}}$, and the corresponding measured terahertz output power, denoted by $P_{\mathrm{THz}}$.

The generated terahertz signal is then propagated over a wireless channel, which comprised two identical WR-3.4 diagonal horn antennas aligned in the line-of-sight (LoS) configuration along their directions of maximum emission with an antenna separation of $15 \mathrm{~mm}$. The estimated free-space propagation loss at this distance, using the Friis transmission formula, is $-25.83 \mathrm{~dB}$. Moreover, this propagation distance is within the wireless transmission range specified by the IEEE 802.15.3d Standard [10] for close-proximity terahertz wireless links [34]-[40].

At the receiver side, the baseband envelope of the received terahertz signal is detected via the SBD and the free space path loss is compensated by a $30 \mathrm{~dB}$ low-noise amplifier (LNA). The amplified signal is then sampled by a $80 \mathrm{GSample/s} \mathrm{digital}$ storage oscilloscope (DSO) for offline DSP using MATLAB as illustrated in Fig. 6. To match the sampling rate at the transmitter side, the recorded data is down-sampled from $80 \mathrm{GSample/s}$ to $64 \mathrm{GSample/s}$ using a down-sampling ratio

TABLE III

THE UTC-PD PHOTO-CURRENT IN MA UNIT AND THE CORRESPONDING MEASURED OUTPUT TERAHERTZ POWER IN $\mathrm{dBM}$ UNIT.

\begin{tabular}{ccccccc}
\hline$I_{\mathrm{ph}}$ & 1 & 2 & 3 & 4 & 5 & 6 \\
\hline$P_{\mathrm{THz}}$ & -28.2 & -22.1 & -18.4 & -15.5 & -13.3 & -12.3 \\
\hline
\end{tabular}




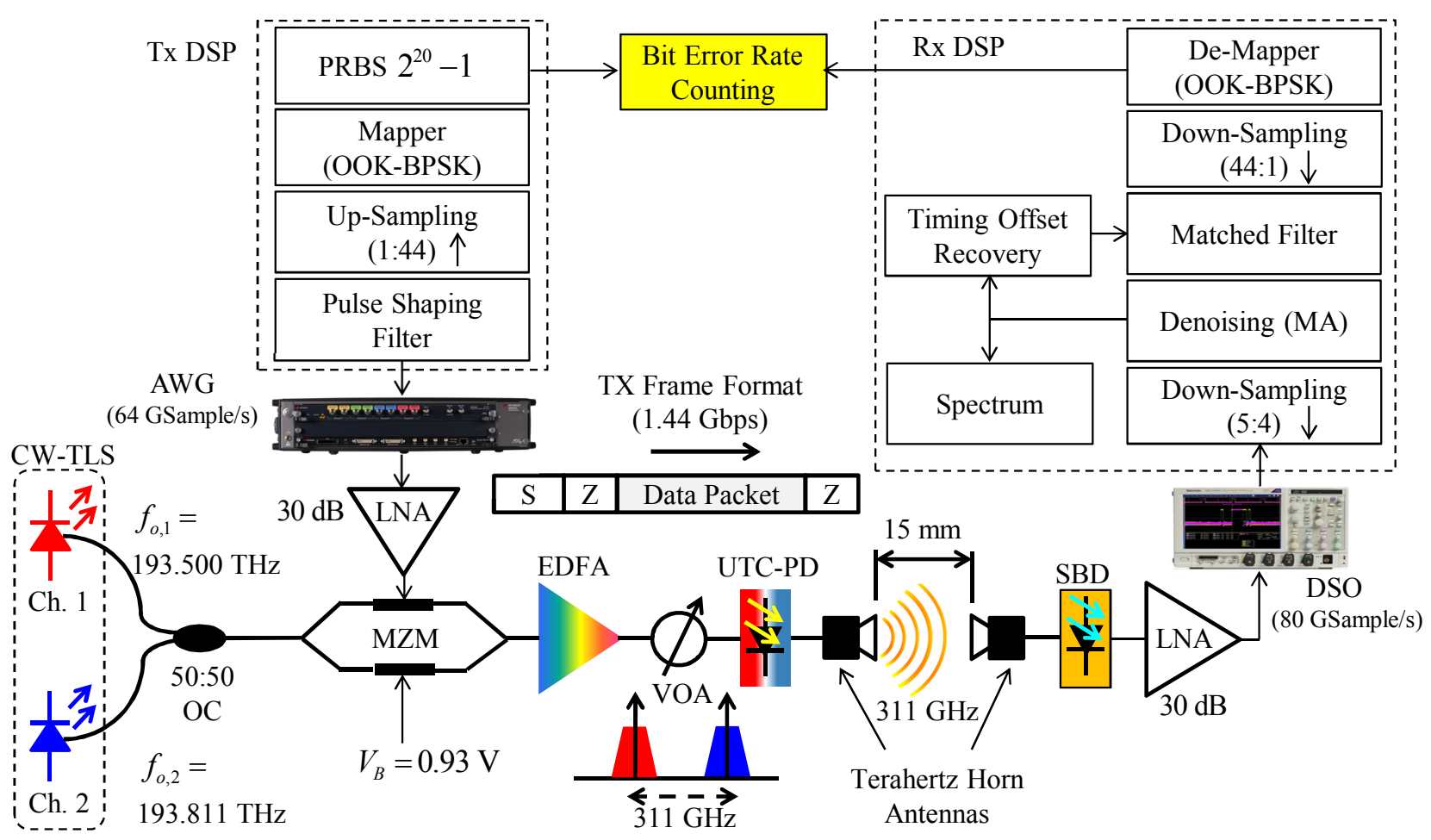

Fig. 6. Experimental setup of the terahertz photonic communications link based on optical heterodyning. TLS: tunable laser source. AWG: arbitrary waveform generator. LNA: low-noise amplifier. MZM: Mach-Zhender modulator. EDFA: erbium doped fiber amplifier. VOA: variable optical attenuator. UTC-PD: unitraveling carrier photodiode. SBD: Schottky barrier diode. LPF: low-pass filter. DSO: digital sampling oscilloscope. MA: moving average. S: synchronization word. D: data payload. Z: zero-padding sequence. The maximum sampling rate of the AWG is 65 GSample/s.

of 5:4 to remove the redundant samples. To mitigate the AWGN, a 44-taps moving average (MA) filter is applied to the received signal as a denoising filter. This filter length is equivalent to a sliding window width of the symbol duration, measured in samples, to ensure that the AWGN is averaged over the symbol duration without influencing the information content in adjacent symbols. The synchronization word is then extracted from the denoised signal for timing offset estimation and compensation. The sliding window approach [41] is also adopted for frame-level synchronization [42], which is achieved by estimating the optimum delay that maximizes the cross-correlation of the received sequence and the synchronization word. Figures 7(a) and 8(a) confirm the accuracy of the timing offset estimation and recovery using this algorithm at the receive side.

Figures 7(b) and 8(b) show the received baseband waveforms after denoising and synchronization, at a UTC-PD photo-current of $3 \mathrm{~mA}$ for OOK and BPSK modulation schemes, respectively. Figures 7(c) and 8(c) plot the spectra of these two signals at the same value of the UTC-PD photocurrent. For the OOK spectrum in Fig. 7(c), a 6-dBr carrier component is detected at $f=f_{c}$ due to the non-zero net DC component of the transmitted OOK signal. This relative power level is much lower than the $40 \mathrm{dBr}$ carrier-to-signal power margin allowed by the IEEE 802.15.3d Standard for the unmodulated carrier component in the OOK terahertz signaling mode.

Moreover, as shown in Figs. 7(c) and 8(c), at $I_{\mathrm{ph}}=3 \mathrm{~mA}$, the received signals' spectra comply with the IEEE spectral mask over most of the channelization scheme bandwidth. It should be pointed out that, although the compliance requirements is achieved throughout the waveform design phase, this requirement is partially violated by the received signal spectrum. This partial violation is attributed to the spectral regrowth effects caused by the nonlinearity of the SBD-based envelope detector [43]. However, since these nonlinear effects increase with increasing the photo-current [43], the violation of the compliance condition is less severe for $I_{\mathrm{ph}} \leq 3 \mathrm{~mA}$. Importantly, the full-compliance of the emitted terahertz signal is not affected as these nonlinear distortions occur at the receiver. Moreover, the nonlinear response of SBD-based receivers can be mitigated by employing Kramer-Kronig (KK) processing at the receiver to improve its decoding capabilities [43]-[47].

The BER performance of the RC, BTN and proposed Lorentzian-based pulse shapes are also evaluated under the OOK and BPSK modulation formats, without applying forward error correction (FEC) techniques. The results, which are plotted in Figs. 9(a) and (b), show that the lowest BER performance is achieved by the proposed waveform, regardless of the modulation format. In Fig. 9(a), the OOK-modulated RC and BTN waveforms show similar BER trends for $1 \mathrm{~mA} \leq$ $I_{\mathrm{ph}} \leq 6 \mathrm{~mA}$ with a minimum BER of $5.952 \times 10^{-5}$ and $1.7 \times 10^{-3}$, respectively, achieved at $I_{\mathrm{ph}}=5 \mathrm{~mA}$. However, increasing the photo-current $I_{\mathrm{ph}}$ beyond $5 \mathrm{~mA}$ deteriorates the BER of both waveforms. This effect is attributed to the nonlinearity of the terahertz link [43], [44], which induces 

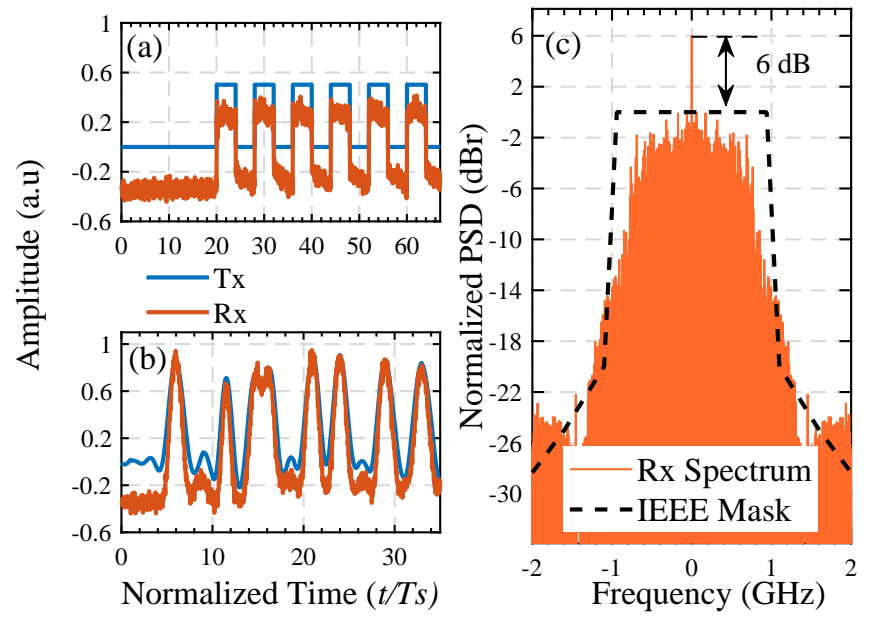

Fig. 7. Signals and spectra at the receiver side for OOK modulation. (a) Segment of the synchronization word, (b) segment of the data payload, and (c) spectrum of the received signal after de-noising. The UTC-PD photo-current is $3 \mathrm{~mA}$.

harmonic and inter-modulation distortion components that grow with increasing photo-current and hence dominate the desired signal. Figure 9(b) shows that the BPSK-modulated RC and BTN waveforms also follow similar BER trends for 1 $\mathrm{mA} \leq I_{\mathrm{ph}} \leq 5 \mathrm{~mA}$ with a minimum BER of $1.4 \times 10^{-3}$ and $2.5 \times 10^{-3}$, respectively, both achieved at $I_{\mathrm{ph}}=5 \mathrm{~mA}$. An error-free transmission is observed for both waveforms when $I_{\mathrm{ph}}$ is increased beyond $5 \mathrm{~mA}$.

On the other hand, for the proposed waveform, the BER decreases monotonically with increasing the photo-current from $1 \mathrm{~mA}$ to $3 \mathrm{~mA}$ for both OOK and BPSK modulation formats. A minimum BER of about $10^{-4}$ and $10^{-3}$ is achieved by this waveform at $I_{\mathrm{ph}}=3 \mathrm{~mA}$ with $\mathrm{OOK}$ and the BPSK modulations, respectively. An error-free transmission is observed when $I_{\mathrm{ph}}$ is increased beyond $3 \mathrm{~mA}$. From the aforementioned observations, it can be concluded that the transmission performance is more sensitive to the SRE rather than the ISI.

\section{CONCLUSION}

In this work, we establish the context of pulse shaping for terahertz communications networks that comply with the technical specifications defined in the IEEE 802.15.3d Standard. An analytical framework is formulated for the design of optimal terahertz envelope waveforms, with an aim to maximize the spectral radiation efficiency under the spectral emission constraints defined in this Standard. Importantly, a waveform design is proposed and compared to the RC and BTN waveforms via numerical analysis and experiments. The proposed waveform shows full compliance with the most stringent spectral mask defined by the IEEE 802.16.3d Standard, where the largest number of densely packed channels can be found. Additionally, this waveform utilizes more than $99 \%$ of the power spectral density admissible by this spectral mask and outperforms the conventional RC and the BTN waveforms by about $35 \%$ in terms of the radiation efficiency, leading
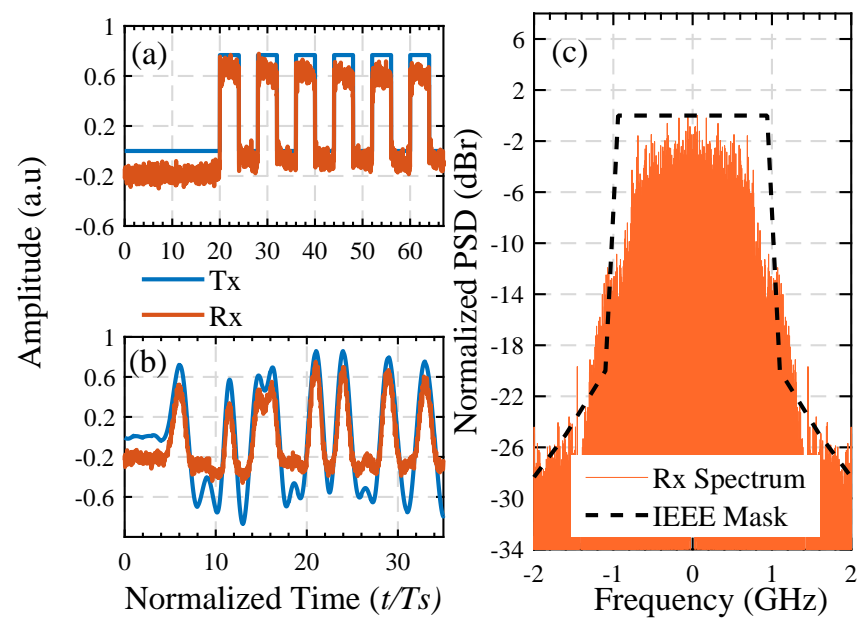

Fig. 8. Signals and spectra at the receiver side for BPSK modulation. (a) Segment of the synchronization word, (b) segment of the data payload, and (c) spectrum of the received signal after de-noising. The UTC-PD photo-current is $3 \mathrm{~mA}$.
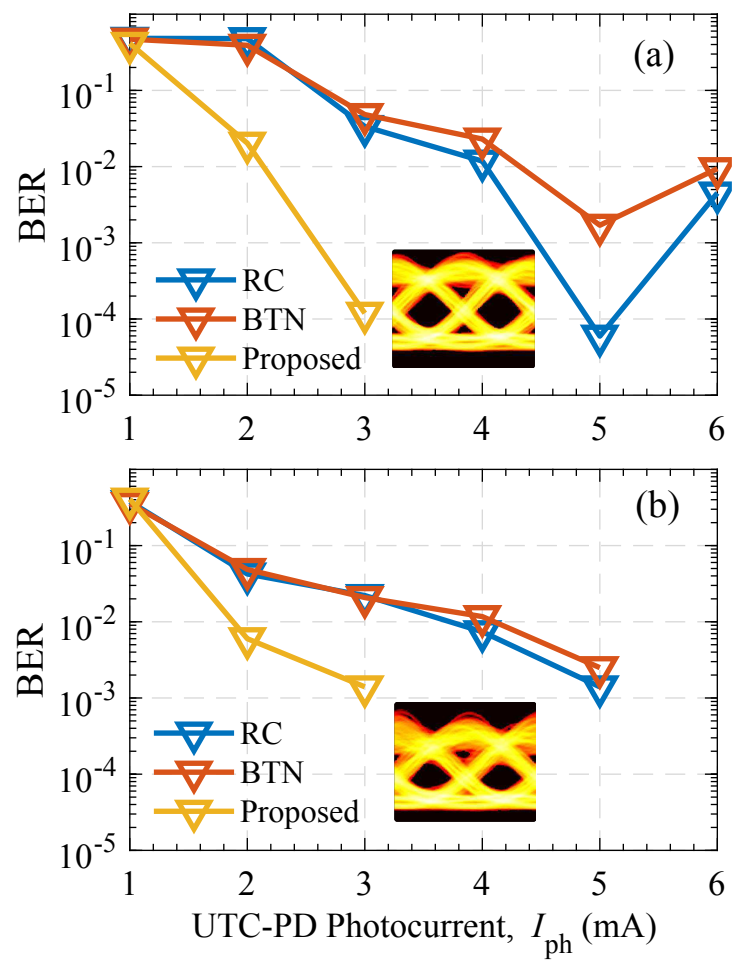

Fig. 9. Bit error rate performance of the proposed waveforms as compared to the RC and the BTN waveform. (a) OOK and (b) BPSK modulation schemes. The eye diagrams are plotted for the proposed waveform obtained at a photocurrent of $3 \mathrm{~mA}$. The line plots are terminated at the points after which the error-free transmission limit is achieved. BER: bit error rate. RC: raisedcosine.

to an improvement of, at least, one order of magnitude in the BER performance. Moreover, experimental results show that the transmission error-free limit of $10^{-12}$ defined by the IEEE 802.15.3d Standard can be achieved using the proposed waveform without employing FEC codes. Additionally, since the bandwidths of all IEEE 802.15.3d emission masks are in- 
teger multiples of $2.16 \mathrm{GHz}$, the proposed waveform presented in this work is applicable to the design and development of standard-compliant terahertz communications systems considering the higher-order channelization schemes.

\section{REFERENCES}

[1] K. Tekbıyık, A. R. Ekti, G. K. Kurt, and A. Görçin, "Terahertz band communication systems: challenges, novelties and standardization efforts," Physical Communication, vol. 35, pp. 1-18, July 2019.

[2] R. E. Floyd and R. H. Spencer, "But will they buy it?" IEEE Potentials, vol. 29 , no. 5 , pp. $14-15,2010$.

[3] S. Y. Shin, "Throughput analysis of IEEE 802.15.4 network under IEEE 802.11 network interference," AEU - International Journal of Electronics and Communications, vol. 67, no. 8, pp. 686-689, 2013.

[4] T. Gigl, F. Troesch, J. Preishuber-pfluegl, and K. Witrisal, "Maximal operating distance estimation for ranging in IEEE 802.15.4a ultra wideband," in The 7th Workshop on Positioning, Navigation and Communication, Dresden, Germany, 2010, pp. 10-18.

[5] J. P.-P. Thomas Gigl, Florian Troesch and K. Witrisal, "Ranging performance of the IEEE 802.15.4a UWB standard under FCC/CEPT regulations," Journal of Electrical and Computer Engineering, vol. 2012, pp. $1-9,2012$.

[6] W. Du, D. Navarro, and F. Mieyeville, "Performance evaluation of IEEE 802.5.4 sensor networks in industrial applications," International Journal of Communication Systems, vol. 28, no. 10, pp. 1657-1674, 2015.

[7] P. Tuset-Peiró, F. Vázquez-Gallego, J. Muñoz, T. Watteyne, J. AlonsoZarate, and X. Vilajosana, "Experimental interference robustness evaluation of SUN-OFDM physical layers," Electronics, vol. 8, no. 9, pp. $1-16$.

[8] R. Dalce, A. V. D. Bossche, T. Val, R. Dalce, A. V. D. Bossche, and T. Val, "An experimental performance study of an original ranging protocol based on an IEEE 802.15.4a UWB testbed," in 2014 IEEE International Conference on Ultra-WideBand (ICUWB), September 2014 , pp. 7-12.

[9] D. D. Guglielmo, B. A. Nahas, S. Duquennoy, T. Voigt, and G. Anastasi, "Analysis and experimental evaluation of IEEE802.15.4e TSCH CSMACA algorithm," IEEE Transactions on Vehicular Technology, vol. 66, no. 2, pp. 1573-1588, 2016.

[10] "IEEE Standard for High Data Rate Wireless Multi-Media Networks-Amendment 2: $100 \mathrm{~Gb} / \mathrm{s}$ Wireless Switched Point-to-Point Physical Layer," IEEE Std 802.15.3d-2017 (Amendment to IEEE Std 802.15.3-2016 as amended by IEEE Std 802.15.3e-2017), pp. 1-55, 2017.

[11] H. J. Song, K. Ajito, Y. Muramoto, A. Wakatsuki, T. Nagatsuma, and N. Kukutsu, "Uni-travelling-carrier photodiode module generating $300 \mathrm{GHz}$ power greater than $1 \mathrm{~mW}$," IEEE Microwave and Wireless Components Letters, vol. 22, no. 7, pp. 363-365, 2012.

[12] V. K. Chinni, P. Latzel, M. Zégaoui, C. Coinon, X. Wallart, E. Peytavit, J. F. Lampin, K. Engenhardt, P. Szriftgiser, M. Zaknoune, and G. Ducournau, "Single-channel $100 \mathrm{Gbit} / \mathrm{s}$ transmission using III-V UTC-PDs for future IEEE 802.15.3d wireless links in the $300 \mathrm{GHz}$ band," Electronics Letters, vol. 54, no. 10, pp. 638-640, 2018.

[13] H. Shams, M. J. Fice, L. Gonzalez-Guerrero, C. C. Renaud, F. Van Dijk, and A. J. Seeds, "Sub-THz wireless over fiber for frequency band 220-280 GHz," Journal of Lightwave Technology, vol. 34, no. 20, pp. 4786-4793, 2016.

[14] S. Koenig, D. Lopez-Diaz, J. Antes, F. Boes, R. Henneberger, A. Leuther, A. Tessmann, R. Schmogrow, D. Hillerkuss, R. Palmer, T. Zwick, C. Koos, W. Freude, O. Ambacher, J. Leuthold, and I. Kallfass, "Wireless sub-THz communication system with high data rate," Nature Photonics, vol. 7, no. 12, pp. 977-981, 2013.

[15] J. Webber, N. Nishigami, J. Y. Kim, M. Fujita, and T. Nagatsuma, "Terahertz wireless communications using resonant tunnelling diodes with radio-over-fibre," Electronics Letters, vol. 55, no. 17, pp. 949-951, 2019.

[16] S. Jia, X. Yu, H. Hu, J. Yu, P. Guan, F. Da Ros, M. Galili, T. Morioka, and L. K. Oxenløwe, "THz photonic wireless links with 16-QAM modulation in the $375-450 \mathrm{GHz}$ band," Optics Express, vol. 24, no. 21, pp. 23777-23 783, 2016.

[17] G. Ducournau, P. Szriftgiser, A. Beck, D. Bacquet, F. Pavanello, E. Peytavit, M. Zaknoune, T. Akalin, and J. F. Lampin, "Ultrawide-bandwidth single-channel $0.4-\mathrm{THz}$ wireless link combining broadband quasi-optic photomixer and coherent detection," IEEE Transactions on Terahertz Science and Technology, vol. 4, no. 3, pp. 328-337, 2014.
[18] V. K. Chinni, M. Zezaoui, C. Coinon, X. Wallart, E. Pevtavit, J. F. Larnpin, P. Szriftgiser, M. Zaknoune, and G. Ducournau, "Indoor 100 Gbit/s THz data link in the $300 \mathrm{GHz}$ band using fast photodiodes," 2018 25th International Conference on Telecommunications, ICT 2018, pp. 288-290, 2018.

[19] M. Fujishima, "Key technologies for THz wireless link by silicon CMOS integrated circuits," Photonics, vol. 5, no. 4, pp. 1-17, 2018.

[20] I. Dan, G. Ducournau, S. Hisatake, P. Szriftgiser, R. P. Braun, and I. Kallfass, "A terahertz wireless communication link using a superheterodyne approach," IEEE Transactions on Terahertz Science and Technology, vol. 10, no. 1, pp. 32-43, 2020.

[21] M. Shehata, K. Wang, J. Webber, F. Masayuki, T. Nagatsuma, and W. Withayachumnankul, " Pulse shaping for IEEE 802.15.3d standard compliant terahertz communications," in 2020 Third International Workshop on Mobile Terahertz Systems (IWMTS), July 2020, pp. 1-5.

[22] A. Adalan, M. Fischer, T. Gigl, K. Witrisal, A. L. Scholtz, and C. F. Mecklenbr, "Ultra-wideband radio pulse shaping filter design for IEEE 802.15.4a transmitter," in 2009 IEEE Wireless Communications and Networking Conference, April 2009, pp. 1-6.

[23] R. Schmogrow, M. Winter, M. Meyer, D. Hillerkuss, B. Nebendahl, J. Meyer, M. Dreschmann, M. Huebner, J. Becker, C. Koos, W. Freude, and J. Leuthold, "Real-time Nyquist pulse modulation transmitter generating rectangular shaped spectra of $112 \mathrm{Gbit} / \mathrm{s}$ 16QAM signals," Advanced Photonics, OSA Technical Digest (CD), vol. 1, no. I, pp. 1516, 2011.

[24] R. Schmogrow, D. Hillerkuss, M. Dreschmann, M. Huebner, M. Winter, J. Meyer, B. Nebendahl, C. Koos, J. Becker, W. Freude, and J. Leuthold, "Real-time software-defined multiformat transmitter generating 64QAM at 28 GBd," IEEE Photonics Technology Letters, vol. 22, no. 21, pp. 1601-1603, 2010.

[25] M. Fellows, C. Baylis, L. Cohen, and R. J. Marks Ii, "Real-time load impedance optimization for radar spectral mask compliance and power efficiency," IEEE Transactions on Aerospace and Electronic Systems, vol. 51, no. 1, pp. 591-599, 2015.

[26] M. Fellows, J. Barlow, C. Baylis, J. Barkate, and R. J. Marks, "Designing power amplifiers for spectral compliance using spectral mask load-pull measurements," in Proceedings, IEEE Topical Conference on Power Amplifiers for Wireless and Radio Applications, January 2015, pp. 1-3.

[27] S. T. Abraha, C. Okonkwo, H. Yang, D. Visani, Y. Shi, H. D. Jung, E. Tangdiongga, and T. Koonen, "Performance evaluation of IR-UWB in short-range fiber communication using linear combination of monocycles," Journal of Lightwave Technology, vol. 29, no. 8, pp. 1143-1151, 2011.

[28] A. Kumar, M. Magarini, and S. Bregni, "Improving GFDM symbol error rate performance using better than Nyquist pulse shaping filters," IEEE Latin America Transactions, vol. 15, no. 7, pp. 1244-1249, 2017.

[29] P. A. Haigh, P. Chvojka, S. Zvánovec, Z. Ghassemlooy, and I. Darwazeh, "Analysis of Nyquist pulse shapes for carrierless amplitude and phase modulation in visible light communications," Journal of Lightwave Technology, vol. 36, no. 20, pp. 5023-5029, 2018.

[30] N. C. Beaulieu, C. C. Tan, and M. O. Damen, "A "better than" Nyquist pulse," IEEE Communications Letters, vol. 5, no. 9, pp. 367-368, 2001.

[31] H. Farès, D. Glattli, Y. Louët, J. Palicot, P. Roulleau, H. Farès, D. Glattli, Y. Louët, J. Palicot, P. Roulleau, and P. Spectrum, "Power spectrum density of single side band CPM using Lorentzian frequency pulses," IEEE Wireless Communications Letters, vol. 6, no. 6, pp. 786-789, 2017.

[32] D. C. Pace, M. Shi, J. E. Maggs, G. J. Morales, and T. A. Carter, "Exponential frequency spectrum and Lorentzian pulses in magnetized plasmas," Physics of Plasmas, vol. 15, no. 12, pp. 1-13, 2008.

[33] M. Bobula, A. Prokeš, and K. Daněk, "Nyquist filters with alternative balance between time- and frequency-domain parameters," Eurasip Journal on Advances in Signal Processing, vol. 2010, pp. 8-10, 2010.

[34] Y. Kado, M. Shinagawa, H. J. Song, and T. Nagatsuma, "Close proximity wireless communication technologies using shortwaves, microwaves, and sub-terahertz waves," Progress in Electromagnetics Research Symposium, vol. 1, pp. 767-772, 2010.

[35] A. Hirata and J. Hirokawa, "Absorber integrated planar slot array antenna for suppression of multiple reflection in 120-GHz-band close-proximity wireless system," vol. E101C, no. 10, pp. 791-800, 2018.

[36] A. Hirata and Hirokawa, Jiro, "Terahertz absorber technologies for closeproximity wireless system," in 2018 Asia-Pacific Microwave Conference (APMC), November 2018, pp. 434-436.

[37] T. Nagatsuma, K. Oogimoto, Y. Inubushi, and J. Hirokawa, "Ultra-stable near-field terahertz communications," in 2016 Progress in Electromagnetic Research Symposium (PIERS), Shanghai, China, August 2016, pp. 3916-3916. 
[38] Tadao Nagatsuma, Kazuki Oogimoto, Yuki Inubushi, and Jiro Hirokawab, "Practical considerations of terahertz communications for short distance applications," Nano Communication Networks, vol. 10, pp. 112, 2016.

[39] Y. Nishida, N. Nishigami, S. Diebold, J. Kim, M. Fujita, and T. Nagatsuma, "Terahertz coherent receiver using a single resonant tunneling diode," Scientific Reports, vol. 9, no. 1, pp. 1-3, 2019.

[40] S. Diebold, K. Nishio, Y. Nishida, J. Y. Kim, K. Tsuruda, T. Mukai, M. Fujita, and T. Nagatsuma, "High-speed error-free wireless data transmission using a terahertz resonant tunneling diode transmitter and receiver," vol. 52, no. 24, pp. 1999-2001, 2016.

[41] J. L. Massey, "Optimum frame synchronization," IEEE Transactions on Communications, vol. 20, no. 2, pp. 115-119, 1972.

[42] S. Pfletschinger, M. Navarro, and P. Closas, "Frame synchronization for next generation uplink coding in deep space communications," in 2015 IEEE Global Communications Conference (GLOBECOM), December 2015, pp. 1-6.

[43] T. Harter, C. Füllner, J. N. Kemal, S. Ummethala, J. L. Steinmann, M. Brosi, J. L. Hesler, E. Bründermann, A. S. Müller, W. Freude, S. Randel, and C. Koos, "Generalized Kramers-Kronig receiver for coherent terahertz communications," Nature Photonics, vol. 14, no. 10, pp. 601-606, 2020.

[44] A. N. M. Ecozzi, "Kramers - Kronig receivers," Advances in Optics and Photonics, vol. 11, no. 3, pp. 480-517, 2019.

[45] S. An, Q. Zhu, J. Li, Y. Ling, and Y. Su, "112-Gb/s SSB 16-QAM signal transmission over $120-\mathrm{km}$ SMF with direct detection using a MIMOANN nonlinear equalizer," Optics Express, vol. 27, no. 9, pp. 12794 $12805,2019$.

[46] Z. Li, M. S. Erkilinç, K. Shi, E. Sillekens, L. Galdino, B. C. Thomsen, P. Bayvel, and R. I. Killey, " $168 \mathrm{~Gb} / \mathrm{s} / \lambda$ direct-detection 64-QAM SSB Nyquist-SCM transmission over $80 \mathrm{~km}$ uncompensated SSMF at 4.54 b/s/Hz net ISD using a Kramers-Kronig receiver," European Conference on Optical Communication, ECOC, vol. 2017-Sept., pp. 1-3, 2017.

[47] Z. Li, M. S. Erkilinc, K. Shi, E. Sillekens, L. Galdino, B. C. Thomsen, P. Bayvel, and R. I. Killey, "SSBI mitigation and the Kramers-Kronig scheme in single-sideband direct-detection transmission with receiverbased electronic dispersion compensation," IEEE/OSA Journal of Lightwave Technology, vol. 35, no. 10, pp. 1887-1893, 2017.

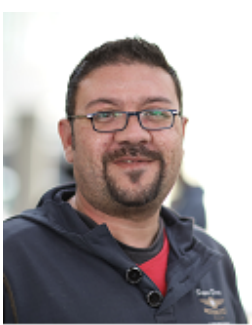

Mohamed Shehata (Senior Member, IEEE) received the B.Sc. and M. Sc. degrees in electronics and communications engineering from Al-Azhar University, Cairo, Egypt, in 2006 and 2012, respectively. He is currently working toward the $\mathrm{PhD}$ degree in electrical engineering with the TeraHertz Engineering Laboratory in the School of Electrical and Electronic Engineering, Faculty of Engineering, Computer and Mathematical Sciences, The University of Adelaide. His research interests include, but not limited to, terahertz communications, terahertz photonics, microwave photonics and radio over fiber (RoF) communications.

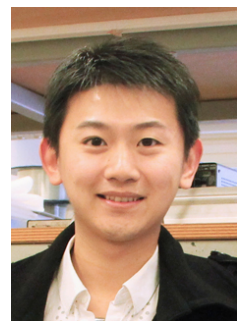

Ke Wang (Member, IEEE) received the B.Sc. degree from the Huazhong University of Science and Technology, Wuhan, China, in 2009, and the Ph.D. degree in electrical and electronic engineering from the University of Melbourne, Melbourne, Vic., Australia, in 2014.

$\mathrm{He}$ is currently an Australian Research Council Discovery Early Career Researcher Award Fellow at the Centre for Neural Engineering, Department of Electrical and Electronic Engineering, University of Melbourne. He is also a Visiting Assistant Professor at Stanford University, Stanford, CA, USA. He has authored or coauthored more than 70 papers in peer-reviewed journals and conferences. His current research interests include silicon photonics integration, optical wireless technology, high-speed personal area networks, and optical interconnects. $\mathrm{He}$ received the IEEE Photonics Society Postgraduate Student Fellowship, the Marconi Society Paul Baran Young Scholar Fellow- ship, the ARC DECRA Fellowship, and the Victoria Fellowship.

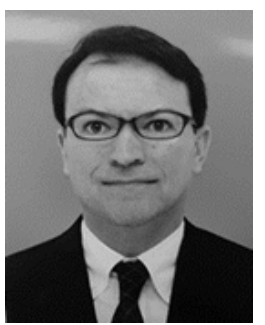

Julian Webber (Senior Member, IEEE) received the $\mathrm{Ph} . \mathrm{D}$. degree in Wireless Communications from the University of Bristol, UK in 2004 after working on DSP and ASIC design at Texas Instruments. He was employed from 2001-07 as a Research Fellow at University of Bristol implementing real-time MIMO and continued with wireless research at Hokkaido University, Japan. He was engaged as a Research Scientist at the Advanced Telecommunications Research Institute (ATR) in Kyoto from 2012-2018 and is currently a specially appointed Assistant Professor in Information Photonics at Osaka University. His current research interests cover wireless and photonic communications systems.

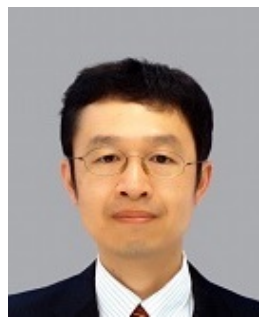

Masayuki Fujita (Member, IEEE) received the $\mathrm{Ph} . \mathrm{D}$. degree from Yokohama National University, Yokohama, Japan, on ultrasmall and ultralowthreshold microdisk lasers, in 2002. Subsequently, he joined the Department of Electronic Science and Engineering at Kyoto University and initiated research on photonic crystals, including spontaneous emission control in photonic crystals and highefficiency light extraction in light-emitting diodes and silicon light emitters. Next, he moved to Osaka University and was appointed the Research Director of the strategic basic research program CREST, "development of terahertz integrated technology platform through fusion of resonant tunneling diodes and photonic crystals" of the Japan Science and Technology Agency.

$\mathrm{He}$ is currently an Associate Professor with the Graduate School of Engineering Science, Osaka University, Toyonaka, Japan. His research interests include terahertz materials and devices, photonic nanostructures and microstructures, and their applications. He is a member of the Japan Society of Applied Physics (JSAP), the Laser Society of Japan, the Institute of Electronics, Information and Communication Engineers, Japan, and the Japanese Photochemistry Association. From 1999 to 2002 and from 2003 to 2006, he was a Research Fellow of the Japan Society for the Promotion of Science (JSPS). He currently serves as an Associate Editor for Applied Physics Express, and a chair of the Technical Group on Terahertz Application Systems of Information and Communication Engineers (IEICE), Japan. 


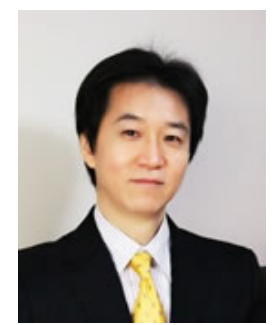

Tadao Nagatsuma (Fellow, IEEE) received the B.S., M.S., and Ph.D. degrees in electronic engineering from Kyushu University, Fukuoka, Japan, in 1981, 1983, and 1986, respectively. In 1986, he joined the Electrical Communications Laboratories, Nippon Telegraph and Telephone Corporation (NTT), Atsugi, Kanagawa, Japan. From 1999 to 2002, he was a Distinguished Technical Member with NTT Telecommunications Energy Laboratories. From 2003 to 2007, he was a Group Leader with NTT Microsystem Integration Laboratories and was an NTT Research Professor from 2007 to 2009. Since 2007, he has been with Osaka University, Toyonaka, Osaka, Japan, where he is currently a Professor with the Division of Advanced Electronics and Optical Science, Department of Systems Innovation, Graduate School of Engineering Science. His research interests include ultrafast electronics and millimeter-wave and terahertz photonics. Dr. Nagatsuma is a Fellow of the Institute of Electronics, Information and Communication Engineers (IEICE), Japan, and a Fellow of the Electromagnetics Academy. He is currently a Track Editor for the IEEE PHOTONICS TECHNOLOGY LETTERS and the IEEE TRANSACTIONS ON TERAHERTZ SCIENCE AND TECHNOLOGY, and a Vice President of the IEICE and the Terahertz Systems Consortium. He was the recipient of numerous awards including the 1989 IEICE Young Engineers Award, the 1992 IEEE Andrew R. Chi Best Paper Award, the 1997 Okochi Memorial Award, the 1998 Japan Microwave Prize, the 2000 Ministers Award of the Science and Technology Agency, the 2002 and 2011 Asia-Pacific Microwave Conference Prize, the 2004 Yokosuka Research Park Award, the 2006 Asia-Pacific Microwave Photonics Conference Award, the 2006 European Microwave Conference Prize, the 2007 Achievement Award presented by the IEICE, the 2008 Maejima Award, the 2011 Recognition from Kinki Bureau of Telecommunications, Ministry of Internal Affairs and Communications, the 2011 Commendation for Science and Technology by the Ministry of Education, Culture, Sports, Science and Technology, the 2014 IEEE Tatsuo Ito Award, and the 2020 Distinguished Achievement and Contributions Award by the IEICE.

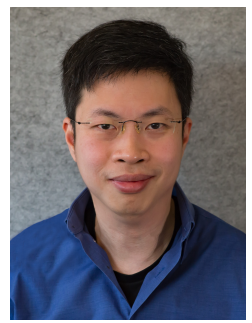

Withawat Withayachumnankul (Senior Member, IEEE) completed bachelor's and master's degrees in electronic engineering from King Mongkut's Institute of Technology Ladkrabang (KMITL), Thailand, in 2001 and 2003, and a doctorate degree in electrical engineering with a Dean's Commendation from the University of Adelaide, Australia, in 2010. In 2010, he was awarded a 3-year Australian Research Council (ARC) Postdoctoral Fellowship. In 2015, he was a Research Fellow of the Japan Society for the Promotion of Science (JSPS) at Tokyo Institute of Technology. He has been a Visiting Researcher at Osaka University in recent years. Dr. Withayachumnankul is currently an Associate Professor with the University of Adelaide, and the founding leader of the Terahertz Engineering Laboratory. Currently, he serves as a Track Editor of the IEEE Transactions on Terahertz Science and Technology. Between 2017-2018, he was a Chair of the IEEE South Australia Joint Chapter on Microwave Theory and Techniques (MTT) \& Antennas and Propagation (AP). He has authored and co-authored more than 90 journal publication, and has supervised $6 \mathrm{PhD}$ students to completion, all with commendation. In recent years, he has been a lead investigator for three Australian Research Council (ARC) grants, totalling to over AUD 1M. His research interests include terahertz waveguides, antennas, radar, communications, and metrology. He is the recipient for the IRMMW$\mathrm{THz}$ Society Young Scientist Award 2020. 